\title{
The patient with rhabdomyolysis: Have you considered quail poisoning?
}

\section{THE CASES}

Patient 1: A 62-year-old woman was admitted with nausea, vomiting, weakness and leg muscle pain. The symptoms had begun 7 hours after a meal of fresh roasted quail. She was not taking any medications, had no allergies to food or medications and did not use alcohol, cigarettes or illicit drugs. Her clinical examination revealed normal cardiopulmonary findings and a temperature of $35.2^{\circ} \mathrm{C}$. She had muscle weakness of both legs but no muscle atrophy or loss of voluntary movements; her deep tendon reflexes were normal. She passed dark brown urine without urgency or dysuria. Results of routine hematological tests were normal. Her creatine kinase (CK) level was $5500 \mathrm{U} / \mathrm{L}$ (this increased to $11000 \mathrm{U} / \mathrm{L}$ the next morning) (normally 30-135 U/L for women) with an MB fraction of $<6 \%$; the levels of other muscle enzymes such as lactate dehydrogenase (1231 U/L), aspartate aminotransferase (200 U/L; this increased to $400 \mathrm{U} / \mathrm{L}$ the next day) and alanine aminotransferase (100 U/L) were also elevated. Results of troponin and direct Coombs' tests were negative, and the patient had an elevated myoglobin level (> $500 \mathrm{U} / \mathrm{L})$; total bilirubin levels were normal. Urinalysis revealed myoglobinuria but no hemoglobinuria. The patient was admitted to hospital, and her symptoms were considered to have been caused by quail ingestion. She rested and was hydrated parenterally. After 4 days she was feeling better, with minimal muscle pains and weakness. Her urine returned to normal, and she was discharged free of symptoms. Her biochemical test results remained normal 1 week later.

Patient 2: A 19-year-old man was referred to hospital for pleuritic chest discomfort and myalgias. He had first experienced diffuse myalgias 24 hours earlier, and these later localized at his chest. His symptoms began about 3 hours after eating a meal that included fresh roasted quail; before eating, he had visited a gym for his usual exercise program. His medical history was unremarkable, and he was taking no medications. His urine was dark brown with myoglobinuria. Hematological test results were normal. His CK level was $1520 \mathrm{U} / \mathrm{L}$ (normally 55-170 U/L for men) with an $\mathrm{MB}$ fraction of $<5 \%$. His myoglobin level $(580 \mathrm{U} / \mathrm{L})$ was also elevated; his aminotransferase level, electrocardiogram and chest radiograph were normal. The patient was hydrated for 2 days and then discharged. One week later, he remained free of symptoms and his biochemical test results were normal.

Patient 3: A 40-year-old man was admitted with diffuse muscle pain in his shoulders and legs and vomiting and nausea during the previous 3 days. He mentioned eating quail at dinner about 8 hours before his symptoms began and engaging in intense muscular exertion the next morning. His clinical examination revealed muscle weakness without any other neurological signs, including numbness or muscular stiffness. The laboratory test results were indicative of rhabdomyolysis, showing mildly increased levels of aminotransferases and lactate dehydrogenase and an elevated CK level (1850 U/L with an MB of fraction < $2 \%)$ and myoglobin level (610 U/L). He did not report any previous illness or relevant family history. Urinalysis revealed myoglobinuria. None of the other members of the dinner party were referred to the hospital or reported any symptoms. The patient was hydrated and treated with bed rest for 3 days and then discharged.

$\mathrm{R}_{\mathrm{t}}^{\mathrm{h}}$ habdomyolysis is an extreme form of myositis, whereby inflamed muscle cells leak their contents into the circulation. This leakage can result in electrolyte abnormalities, acidosis, clotting disorders, hypo$\stackrel{0}{a}$ volemia, myoglobinuria and acute renal failure. Physical symptoms include muscular weakness, swelling, pain, cramping and darkened or teacoloured urine.

One of the most reliable methods of assessing muscular damage is to check for increases in blood serum levels of creatine kinase, which is located in the sarcolemma and mitochondrial intermembrane space of healthy muscle cells. Other laboratory indicators of rhabdomyolysis include elevated serum myoglobin levels, the presence of myoglobinuria and mild increases in aminotransferase and lactate dehydrogenase levels.

Rhabdomyolysis can be caused by a number of factors, including trauma, such as crash syndrome, hyperthermia, infections or other health disorders. Non-traumatic causes include the use of drugs such as statins and strenuous exercise (the latter usually involve people who are inexperienced in exercise and uneducated in fitness and health principles, dehydrated, heat stressed or taking drugs or who are military recruits in basic training).

Several food-related causes of acute rhabdomyolysis have been recognized, including fish (Haff 


\section{Practice}
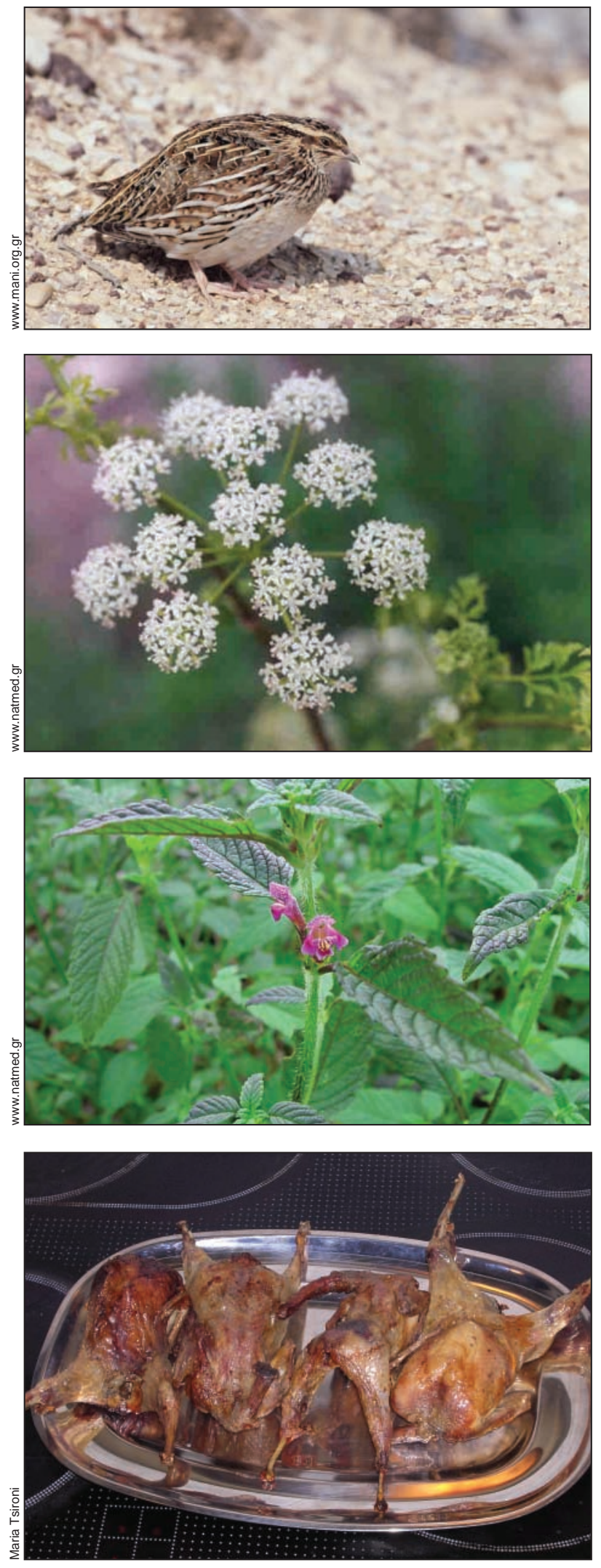

riod of the European common quail, Coturnix coturnix (Fig. 1). Coturnism has been recognized since antiquity; the Old Testament mentions the group poisoning of the Jewish people during the Exodus (Numbers:11).

Clinical symptoms of coturnism usually start 1-9 hours after the bird has been ingested. Symptoms of rhabdomyolysis predominate and neurological features are absent, in contrast to other types of food-toxin poisoning. Unusual smell or taste does not help in identifying toxic food, and cooking methods cannot detoxify a bird capable of causing the syndrome. The relation between quantity consumed and the possibility of becoming ill has not been identified. However, muscular exertion before or after the meal is known to aggravate and accelerate the manifestations, ${ }^{1}$ as may have happened with 2 of our patients. The symptoms of patient 2 , who had visited a gym before the meal and did not take supplements or other drugs, were initially misdiagnosed as indicating an embolic event. Although symptoms may be severe, most cases, such as those reported here, are benign and of short duration. None of our patients experienced headache, a lower level of consciousness or meningism. No muscle stiffness was present.

As no other cause of rhabdomyolysis was evident, we believe that quail consumption was the culprit. All 3 cases occurred in autumn, the quail migration period. Previous reports have speculated that the syndrome is caused by a toxin or alkaloid that has a curare-like action and nicotine effects on autonomic ganglia and which is contained in seeds eaten by the quail, most likely from hemlock (Conium maculatum $)^{2}$ (Fig. 2) or other plants (e.g., red hempnettle, or Galeopsis ladanum) $)^{3,4}$ (Fig. 3). Coniine, the most important alkaloid of $C$. maculatum, can be lethal in a dose of $150 \mathrm{mg},{ }^{4}$ but in smaller doses it produces neurotoxic effects, acute rhabdomyolysis and acute renal failure. ${ }^{5}$ However, coniine triggers clinical manifes- tations only in sensitive people; personal susceptibility seems to be a factor common to coturnism and Haff disease. ${ }^{5}$ The patients described here shared their meals (Fig. 4) with other people who were not affected. Although some investigations have suggested that susceptibility runs in families, ${ }^{1}$ others have excluded a pre-existing enzyme defect as a cause. ${ }^{6}$ Our patients did not report any previous attacks or family histories of such events.

Coturnism is a rare cause of acute rhabdomyolysis, and it should be considered in a geographically and temporally appropriate situation (e.g., the Mediterranean in the fall) where patients experience unexplained rhabdomyolysis, especially if it occurs in an epidemic. Once other causes are ruled out, treatment is mostly supportive, with appropriate volume replacement, urinary alkalinization and aggressive diuresis or hemodialysis if required.

\section{Maria Tsironi}

Panagiotis Andriopoulos

Eytixia Xamodraka

Spyros Deftereos

Athanasios Vassilopoulos

Giorgos Asimakopoulos

Athanasios Aessopos

Department of Internal Medicine

University of Athens Medical School

"Laiko" Hospital

Athens, Greece

This article has been peer reviewed

\section{References}

1. Ouzounellis T. Some notes on quail poisoning. FAMA 1970;211(7):1186-7.

2. Frank AA, Reed WM. Comparative toxicity of coniine, an alkaloid of $\mathrm{Co}^{-}$ nium maculatum (poison hemlock), in chickens, quails, and turkeys. Avian Dis 1990;34(2):433-7.

3. Aparicio $\mathrm{R}$, Onate $\mathrm{JM}$, Arizcun $\mathrm{A}, \mathrm{Al}$ varez T, Alba A, Cuende JI, et al. [Epidemic rhabdomyolysis due to the eating of quail. A clinical, epidemiological and experimental study]. Med Clin (Barc) 1999;112(4):143-6.

4. Kennedy WB, Grivetti EL. Toxic quail: a cultural-etiological investigation of coturnism. Ecol Food Nutr 1980;9:15-42.

5. Moffat AC. Conium maculatum. In: Moffat AC, editor. Clarke's isolation and identification of drugs. London: The Pharmaceutical Press; 1986. p. 493.

6. Papadimitriou A, Hadjigeorgiou GM, Tsairis P, Papadimitriou E, Ouzounelli C, Ouzounellis T. Myoglobinuria due to quail poisoning. Eur Neurol 1996;36(3):142-5. 